\title{
Thermodynamic derivations of conditions for chemical equilibrium and of Onsager reciprocal relations for chemical reactors
}

\author{
Gian Paolo Beretta ${ }^{a}$ \\ Università di Brescia, via Branze 38, Brescia, I-25123, Italy \\ Elias P. Gyftopoulos ${ }^{\text {b) }}$ \\ Massachusetts Institute of Technology, Cambridge, Massachusetts 02139
}

(Received 2 March 2004; accepted 6 April 2004)

\begin{abstract}
For an isolated chemical reactor, we derive the conditions for chemical equilibrium in terms of either energy, volume, and amounts of constituents or temperature, pressure, and composition, with special emphasis on what is meant by temperature and chemical potentials as the system proceeds through nonequilibrium states towards stable chemical equilibrium. For nonequilibrium states, we give both analytical expressions and pictorial representations of the assumptions and implications underlying chemical dynamics models. In the vicinity of the chemical equilibrium state, we express the affinities of the chemical reactions, the reaction rates, and the rate of entropy generation as functions of the reaction coordinates and derive Onsager reciprocal relations without recourse to statistical fluctuations, time reversal, and the principle of microscopic reversibility. (C) 2004 American Institute of Physics. [DOI: 10.1063/1.1756576]
\end{abstract}

\section{INTRODUCTION}

In authoritative discussions ${ }^{1-3}$ of chemical equilibrium among $r$ constituents of a system $A$, the condition of equilibrium in the presence of one chemical reaction mechanism is presumably shown to be

$$
\sum_{i=1}^{r} \nu_{i} \mu_{i}\left(U, V, n_{1}, n_{2}, \ldots, n_{r}\right)=0,
$$

where $\nu_{i}$, for $i=1,2, \ldots, r$, is the $i$ th stoichiometric coefficient of the chemical reaction mechanism

$$
\sum_{i=1}^{r} \nu_{i} A_{i}=0 .
$$

$A_{i}$ denotes the $i$ th constituent, $\mu_{i}$ the chemical potential of the $i$ th constituent, $U$ the energy, $V$ the volume, $n_{1}, n_{2}, \ldots, n_{r}$ the amounts of constituents given by the relations

$$
n_{i}=n_{i a}+\nu_{i} \varepsilon \quad \text { for } i=1,2, \ldots, r,
$$

and $n_{i a}$, for $i=1,2, \ldots, r$, the amount of the $i$ th constituent for which the value of the reaction coordinate $\varepsilon$ is equal to zero.

For given values of $U, \quad V, n_{1 a}, n_{2 a}, \ldots, n_{r a}$, $\nu_{1}, \nu_{2}, \ldots, \nu_{r}$, condition (1) yields the value $\varepsilon_{0}$ for which the system is in the chemical equilibrium or stable equilibrium state. Thus, at chemical equilibrium, the amounts of constituents are given by the relations

$$
n_{i 0}=n_{i a}+\nu_{i} \varepsilon_{0} \quad \text { for } i=1,2, \ldots, r
$$

and the corresponding mole fractions or composition by the relations

\footnotetext{
a)Electronic mail: beretta@unibs.it

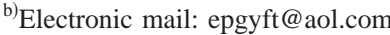

$$
y_{i 0}=\frac{n_{i a}+\nu_{i} \varepsilon_{0}}{n_{a}+\nu \varepsilon_{0}} \text { for } i=1,2, \ldots, r,
$$

where $n_{a}=\sum_{i=1}^{r} n_{i a}$ and $\nu=\sum_{i=1}^{r} \nu_{i}$.

In the discussions just cited, ${ }^{1-3}$ it is also stated that condition (1) results from the requirement that, for an isolated system, the value of the sum $\sum_{i=1}^{r} \mu_{i}(U, V, \mathbf{n}) d n_{i}$ at the chemical equilibrium state must be zero for any variations of the amounts of constituents compatible with the stoichiometry of the reaction mechanism, where $\mathbf{n}=\left\{n_{1}, n_{2}, \ldots, n_{r}\right\}$ denotes the amounts of the $r$ constituents.

Even though experience shows that condition (1) leads to results consistent with observations, its derivation and meaning are problematic. According to the second law of thermodynamics, ${ }^{4}$ an isolated system with one or more chemical reactions, and given values of $U, V$, $n_{1 a}, n_{2 a}, \ldots, n_{r a}$, admits one and only one stable equilibrium state. To that state corresponds a unique composition. Any composition that deviates from that of the stable equilibrium state corresponds to a state that is not stable equilibrium and, therefore, no chemical potentials can be defined. So what functions $\mu_{i}\left(U, V, n_{1}, n_{2}, \ldots, n_{r}\right)$ should be used in condition (1) in order to find $\varepsilon_{0}$ and the chemical equilibrium state?

We investigate this question and find a satisfactory answer for any system $A$ that satisfies the model assumption of what we call a simple system. For such a system, the chemical potentials appearing in condition (1) are those of a surrogate simple system $B$ consisting of the same constituents as $A$, with the difference, however, that all reaction mechanisms are turned off.

We discuss briefly the definition of a simple system in Sec. II, the derivation of conditions for chemical equilibrium of an isolated chemical reactor with $r$ constituents and $c$ chemical reactions in terms of energy, volume, and chemical 
potentials in Sec. III, the derivation of the same conditions in terms of temperature, pressure, and mole fractions in Sec. IV, the rate of entropy generation in the reactor in Sec. V, reciprocal relations in Sec. VI, and our conclusions in Sec. VII.

\section{SIMPLE SYSTEMS}

We define a system as simple $e^{4}$ if it satisfies the following three conditions: (i) it has volume as one of the parameters; (ii) in any of its stable equilibrium states, if it is partitioned into a set of contiguous subsystems in mutual stable equilibrium, the effects of the partitions on the values of all properties are negligible; ${ }^{5}$ and (iii) in any of its stable equilibrium states, the instantaneous switching off or on of one or more internal reaction mechanisms, such as a chemical reaction, causes negligible instantaneous changes in the values of energy, entropy, volume, and amounts of constituents.

In general, either the introduction of partitions or the instantaneous switching on or off of chemical reaction mechanisms or both have definite effects on a system. For example, using the tools of quantum theory, ${ }^{6,7}$ we can show that the switching on of a reaction mechanism requires the switching on of an additional term in the Hamiltonian operator of the system, which affects the functional form of the fundamental relation for stable equilibrium states. Again, using the tools of quantum theory, we can show that the switching off of a reaction mechanism requires the "destruction" of correlations among constituents and, in general, results in a reduction of the value of the entropy. Nevertheless, we can also show that these effects become less and less important, and negligible for all practical purposes, if the value of the amount of each constituent is larger than 10 (Refs. 6 and 7). Hence the simple system model is applicable to most practical systems, including the nanovolume and microvolume scale, with sufficiently large amounts of constituents.

\section{DERIVATION OF CONDITIONS FOR CHEMICAL EQUILIBRIUM}

We consider a simple system $A$ having energy $U$, volume $V$, and constituents $A_{1}, A_{2}, \ldots, A_{r}$ with initial amounts $n_{1 a}, n_{2 a}, \ldots, n_{r a}$, subject to $c$ chemical reaction mechanisms

$$
\sum_{i=1}^{r} \nu_{i}^{(j)} A_{i}=0 \quad \text { for } j=1,2, \ldots, c,
$$

and derive the conditions for $A$ to be in a stable equilibrium or, synonymously, in a chemical equilibrium state, where $\nu_{i}^{(j)}$ are the stoichiometric coefficients of the $j$ th chemical reaction.

In general, the chemical reactor just defined, for each given set of values of $U, V, \mathbf{n}_{a}$, and $\boldsymbol{\nu}$, admits an infinite number of states. However, the laws of thermodynamics require that among these states one and only one be a chemical equilibrium state, and this state has the largest value of the entropy. ${ }^{4}$ We call the latter requirement the highest or largest entropy principle. At the chemical equilibrium state, the values of the amounts of constituents $n_{10}, n_{20}, \ldots, n_{r 0}$ and the corresponding reaction coordinates $\varepsilon_{10}, \varepsilon_{20}, \ldots, \varepsilon_{r 0}$ satisfy the compatibility relations

$$
n_{i 0}=n_{i a}+\sum_{j=1}^{c} \nu_{i}^{(j)} \varepsilon_{j 0} \quad \text { for } i=1,2, \ldots, r
$$

Moreover, the values $U, V, \mathbf{n}_{a}=\left\{n_{1 a}, n_{2 a}, \ldots, n_{r a}\right\}$, and the stoichiometric coefficients $\boldsymbol{\nu}=\left\{\nu_{i}^{(j)}\right.$ for $i=1,2, \ldots, r$ and $j$ $=1,2, \ldots, c\}$ determine uniquely the values of all the properties and quantities that characterize the chemical equilibrium state, including the values of the entropy $S$, each $\varepsilon_{j 0}$, and each $n_{i 0}$. We write the dependences of the latter in the form

$$
\begin{aligned}
& S=S\left(U, V, \mathbf{n}_{a} ; \boldsymbol{\nu}\right), \\
& \varepsilon_{j 0}=\varepsilon_{j 0}\left(U, V, \mathbf{n}_{a} ; \boldsymbol{\nu}\right) \text { for } j=1,2, \ldots, c, \\
& n_{i 0}=n_{i 0}\left(U, V, \mathbf{n}_{a} ; \boldsymbol{\nu}\right) \text { for } i=1,2, \ldots, r .
\end{aligned}
$$

In general, we cannot find the explicit functional forms of Eqs. (8)-(10). For simple systems, however, the problem is somewhat less complicated because we can express chemical equilibrium properties in terms of stable equilibrium properties of a multiconstituent system in which all the chemical reaction mechanisms are inhibited-switched off. To see how this is done, we proceed as follows.

First, we consider a simple system $B$ consisting of the same $r$ types of constituents as system $A$ but with all the chemical reaction mechanisms inhibited-switched off. Of course, $A$ and $B$ are different systems because they are subject to different internal forces and constraints. We assume that $B$ is in a stable equilibrium state with values $U$ of the energy, $V$ of the volume, and $\mathbf{n}=\left\{n_{1}, n_{2}, \ldots, n_{r}\right\}$ of the amounts of the $r$ constituents. We denote the entropy at this stable equilibrium state by the fundamental relation

$$
S_{\text {off }}=S_{\text {off }}(U, V, \mathbf{n})
$$

where we use the subscript "off" to emphasize that all the reaction mechanisms are switched off.

Next, we assume that the chemical reaction mechanisms are instantly switched on, that is, all the reactions defined by the stoichiometric coefficients $\boldsymbol{\nu}$ are no longer inhibited. As a result, we obtain again system $A$. Because in our discussion of chemical reactors we go back and forth between systems $A$ and $B$ by switching off and switching on the chemical reaction mechanisms, we call system $B$ the surrogate system of $A$.

Because the surrogate system $B$ is simple and initially in a stable equilibrium state, immediately after switching on the reaction mechanisms the state of system $A$ has the same values of $S, U, V, n_{1}, n_{2}, \ldots, n_{r}$ as the corresponding values of the stable equilibrium state of $B$. In general, however, this state of $A$ is not stable equilibrium. For example, if $B$ is a quiescent mixture of gasoline vapor and air at room temperature and we activate the reaction mechanisms by a minute spark, we instantly produce a nonequilibrium state of system $A$ in which the reactions are no longer inhibited-the burning 
of the gasoline is proceeding-even though the instantaneous perturbations of the values of $S, U, V, n_{1}, n_{2}, \ldots, n_{r}$ introduced by the spark are negligible.

In other words, it is by virtue of the important key assumption of the simple system model by which reactions can be switched on and off without significantly altering the value of any property that we succeed in expressing properties of stable equilibrium states as well as of a class of nonequilibrium states of a reacting system in terms of the known stable equilibrium properties of nonreacting multicomponent systems.

Among all the states of $A$ that may be obtained from $B$ in the manner just cited, we consider the subset that has given values $U$ and $V$ of the energy and volume, and amounts of constituents that are compatible with given values $n_{1 a}, n_{2 a}, \ldots, n_{r a}$. We denote each of these states by $A_{\varepsilon}$ and recognize that it corresponds to a set of values of the $c$ reaction coordinates $\varepsilon=\left\{\varepsilon_{1}, \varepsilon_{2}, \ldots, \varepsilon_{c}\right\}$ such that

$$
n_{i}=n_{i a}+\sum_{j=1}^{c} \nu_{i}^{(j)} \varepsilon_{j} \quad \text { for } i=1,2, \ldots, r
$$

where all the $n_{i}$ 's are non-negative. Among all the states $A_{\varepsilon}$, the one with the largest entropy is the unique chemical equilibrium state with energy $U$, volume $V$, and amounts of constituents compatible with $\mathbf{n}_{a}$, that is, $\mathbf{n}_{0}=\mathbf{n}_{a}+\boldsymbol{\nu} \cdot \boldsymbol{\varepsilon}_{0}$. We denote the chemical equilibrium state by $A_{\varepsilon_{0}}$.

To prove that indeed state $A_{\varepsilon_{0}}$ corresponds to the largest entropy, we assume that another state $A_{0} \neq A_{\varepsilon_{0}}$ with entropy $S_{0}$, not belonging to the family of states $A_{\varepsilon}$, is the chemical equilibrium state that corresponds to the given values $U, V$, $\mathbf{n}_{a}, \boldsymbol{\nu}$. Then $S_{\varepsilon_{0}}<S_{0}$ because $A_{0}$ has the largest entropy. Now, starting from $A_{0}$, we switch off the chemical reaction mechanisms. Because system $A$ is simple and $A_{0}$ is a stable equilibrium state, the resulting state $B_{0}$ of surrogate system $B$ has the same values $U, V$, and $\mathbf{n}_{0}$ as $A_{0}$ and, in particular, its entropy is $S_{0}$. State $B_{0}$ cannot be stable equilibrium because, if it were, then upon switching the chemical reaction mechanisms back on we would obtain again state $A_{0}$ and conclude that it belongs to the family $A_{\varepsilon}$ contradicting the fact that $A_{\varepsilon_{0}}$ has the largest entropy. On the other hand, if state $B_{0}$ is not stable equilibrium, then the stable equilibrium state of $B$ with values $U, V$, and $\mathbf{n}_{0}$ would have entropy $S>S_{0}$, and switching on the reactions beginning with this state would yield a state in the family $A_{\varepsilon}$ that has entropy $S>S_{0}>S_{\varepsilon_{0}}$, again contradicting our stipulation that $A_{\varepsilon_{0}}$ has the largest entropy. Therefore, if $A_{0}$ is the chemical equilibrium state, it must coincide with state $A_{\varepsilon_{0}}$ because under the specified conditions there is one and only one stable equilibrium state.

Because we can express the entropy $S_{\varepsilon}$ of a state $A_{\varepsilon}$ in terms of the entropy $S_{\text {off }}(U, V, \mathbf{n})$ of the state of the surrogate system to which $A_{\varepsilon}$ corresponds, we can determine the chemical equilibrium entropy $S\left(U, V, \mathbf{n}_{a} ; \boldsymbol{\nu}\right)[$ Eq. (8)] by finding the largest value of $S_{\text {off }}(U, V, \mathbf{n})$.

To find the largest value just cited, we first write the entropy $S_{\varepsilon}$ of a state $A_{\varepsilon}$ in the form

$$
S_{\varepsilon}=S_{\text {off }}\left(U, V, \mathbf{n}_{a}+\boldsymbol{\nu} \cdot \boldsymbol{\varepsilon}\right),
$$

where in the fundamental relation $S_{\text {off }}=S_{\text {off }}(U, V, \mathbf{n})$ we use the shorthand notation $\mathbf{n}_{a}+\boldsymbol{\nu} \cdot \boldsymbol{\varepsilon}$ for the set $\mathbf{n}$ $=\left\{n_{1 a}+\sum_{j=1}^{c} \nu_{1}^{(j)} \varepsilon_{j}, n_{2 a}+\sum_{j=1}^{c} \nu_{2}^{(j)} \varepsilon_{j}, \ldots, n_{r a}+\sum_{j=1}^{c} \nu_{r}^{(j)} \varepsilon_{j}\right\}$. Then we note that in order for $A_{\varepsilon_{0}}$ to be the state of largest entropy among all the states $A_{\varepsilon}$ with given $U, V$, and $\mathbf{n}_{a}$, the values of $\varepsilon_{0}$ must be such that

$$
\left(\frac{\partial S_{\boldsymbol{\varepsilon}}}{\partial \varepsilon_{j}}\right)_{U, V, \mathbf{n}_{a}, \boldsymbol{\nu}, \boldsymbol{\varepsilon}}=0 \text { for } j=1,2, \ldots, c,
$$

where the subscripts $\mathbf{n}_{a}, \boldsymbol{\nu}$, and $\boldsymbol{\varepsilon}$ denote, respectively, that each of the amounts $n_{i a}$, each of the stoichiometric coefficients $\nu_{i}^{(j)}$, and each of the reaction coordinates $\varepsilon_{i}$ that do not appear in the derivative are kept fixed. For $j=1,2, \ldots, c$, from Eq. (13) we find that

$$
\begin{aligned}
\left(\frac{\partial S_{\varepsilon}}{\partial \varepsilon_{j}}\right)_{U, V, \mathbf{n}_{a}, \boldsymbol{\nu}, \boldsymbol{\varepsilon}} & =\sum_{i=1}^{r}\left(\frac{\partial S_{\text {off }}}{\partial n_{i}}\right)_{U, V, \mathbf{n}}\left(\frac{\partial n_{i}}{\partial \varepsilon_{j}}\right)_{\mathbf{n}_{a}, \boldsymbol{\nu}, \boldsymbol{\varepsilon}} \\
& =-\sum_{i=1}^{r} \frac{\mu_{i, \text { off }}}{T_{\text {off }}} \nu_{i}^{(j)} \\
& =Y_{j, \text { off }}\left(U, V, \mathbf{n}_{a}+\boldsymbol{\nu} \cdot \boldsymbol{\varepsilon}\right) \\
& =\sum_{i=1}^{r}\left(-\mu_{i, \text { off }} \tau_{\text {off }}\right) \nu_{i}^{(j)},
\end{aligned}
$$

where $T_{\text {off }}$ is the temperature and $\mu_{i \text {,off }}$ the chemical potential of constituent $i$ of the stable equilibrium state of the surrogate system $B$ that corresponds to $A_{\varepsilon_{0}}, \tau=1 / T$, and in writing Eqs. (15b) and (15d) we use the relations $\left(\partial S / \partial n_{i}\right)_{U, V, \mathbf{n}}$ $=-\mu_{i} / T=-\mu_{i} \tau$ and Eq. (12), and in writing Eq. (15c) we define $Y_{j, \text { off }}=\mathcal{A}_{j, \text { off }} / T_{\text {off }}$ where $\mathcal{A}_{j \text {, off }}=-\Sigma_{i=1}^{r} \nu_{i}^{(j)} \mu_{i, \text { off }}$ is the so-called affinity of the $j$ th reaction, which is clearly a stable equilibrium property of surrogate system $B$.

For finite values of $T_{\text {off }}$, we see from Eqs. (14) and (15) that a set of necessary conditions that relate $U, V, \mathbf{n}_{a}, \boldsymbol{\mu}_{\mathrm{off}}$, $\boldsymbol{\nu}$, and $\boldsymbol{\varepsilon}_{0}$ at chemical equilibrium are

$\sum_{i=1}^{r} \nu_{i}^{(j)} \mu_{i, \text { off }}\left(U, V, \mathbf{n}_{a}+\boldsymbol{\nu} \cdot \boldsymbol{\varepsilon}_{0}\right)=0$ for $j=1,2, \ldots, c$

or, equivalently, $Y_{j, \text { off }}\left(U, V, \mathbf{n}_{a}+\boldsymbol{\nu} \cdot \boldsymbol{\varepsilon}_{0}\right)=0$. In the next section we show that $T$ and $\mu_{i}$ for $i=1,2, \ldots, r$ are also equal to the temperature and chemical potentials of the chemical equilibrium state of system $A$. Each of Eqs. (16) is the chemical equilibrium equation for the corresponding reaction mechanism.

For each given set of values $U, V, \mathbf{n}_{a}$, and $\boldsymbol{\nu}$, Eqs. (16) are $c$ necessary conditions for chemical equilibrium. They may be solved to yield Eqs. (8) to (10) and, therefore, all properties of the chemical equilibrium state. They confirm the statement made earlier to the effect that properties of chemical equilibrium may be expressed in terms of properties of a multiconstituent system with all chemical reaction mechanisms switched off.

For the extremum corresponding to Eqs. (14) to be a relative maximum, it is also necessary that the second-order 
partial differential of $S_{\varepsilon}$ with respect to the $c$ reaction coordinates $\varepsilon_{1}, \varepsilon_{2}, \ldots, \varepsilon_{c}$ be negative. To show that indeed this is the case, we start with Eq. (13) and find that

$$
\begin{aligned}
& \left(d^{2} S_{\boldsymbol{\varepsilon}}\right)_{U, V, \mathbf{n}_{a}, \boldsymbol{\nu}} \\
& =\frac{1}{2} \sum_{j=1}^{c} \sum_{k=1}^{c}\left(\frac{\partial^{2} S_{\boldsymbol{\varepsilon}}}{\partial \boldsymbol{\varepsilon}_{j} \partial \boldsymbol{\varepsilon}_{k}}\right)_{U, V, \mathbf{n}_{a}, \boldsymbol{\nu}} d \boldsymbol{\varepsilon}_{j} d \varepsilon_{k} \\
& =\frac{1}{2} \sum_{j=1}^{c} \sum_{k=1}^{c} \sum_{p=1}^{r} \sum_{q=1}^{r}\left(\frac{\partial^{2} S_{\text {off }}}{\partial n_{p} \partial n_{q}}\right)_{U, V, \mathbf{n}} \nu_{p}^{(j)} \nu_{q}^{(k)} d \varepsilon_{j} d \varepsilon_{k} \\
& =\frac{1}{2} \sum_{p=1}^{r} \sum_{q=1}^{r}\left(\frac{\partial^{2} S_{\text {off }}}{\partial n_{p} \partial n_{q}}\right)_{U, V, \mathbf{n}} \sum_{j=1}^{c} \nu_{p}^{(j)} d \varepsilon_{j} \sum_{k=1}^{c} \nu_{q}^{(k)} d \varepsilon_{k} \\
& =\frac{1}{2} \sum_{p=1}^{r} \sum_{q=1}^{r}\left(\frac{\partial^{2} S_{\text {off }}}{\partial n_{p} \partial n_{q}}\right)_{U, V, \mathbf{n}} d n_{p} d n_{q}<0,
\end{aligned}
$$

where we use $d n_{i}=\sum_{j=1}^{c} \nu_{i}^{(j)} d \varepsilon_{j}$ for $i=1,2, \ldots, r$. The inequality is always satisfied because $S_{\text {off }}$ is the fundamental relation of the surrogate system $B$ and, as such, it is concave with respect to every $n_{i}$ for $i=1,2, \ldots, r$ (Ref. 4 ). Each of the necessary conditions for chemical equilibrium-each of Eqs. (16) — is expressed as a function of energy, volume, and amounts of constituents of the chemical equilibrium state. In the next section we rewrite these conditions in terms of temperature and pressure rather than energy and volume.

For fixed values of $U, V, \mathbf{n}_{a}$, and $\boldsymbol{\nu}$, from Eqs. (13) and (15) we see that each of the functions $S_{\text {off }}$ and $Y_{j, \text { off }}$ for $j$ $=1,2, \ldots, c$ depends solely on the reaction coordinates $\varepsilon_{k}$ for $k=1,2, \ldots, c$. Accordingly, for $j, k=1,2, \ldots, c$, we can write

$$
\frac{\partial^{2} S_{\text {off }}\left(U, V, \mathbf{n}_{a}+\boldsymbol{\nu} \cdot \boldsymbol{\varepsilon}\right)}{\partial \varepsilon_{j} \partial \varepsilon_{k}}=\frac{\partial^{2} S_{\text {off }}\left(U, V, \mathbf{n}_{a}+\boldsymbol{\nu} \cdot \boldsymbol{\varepsilon}\right)}{\partial \varepsilon_{k} \partial \varepsilon_{j}}
$$

or, equivalently,

$$
\left(\frac{\partial Y_{k, \text { off }}}{\partial \boldsymbol{\varepsilon}_{j}}\right)_{U, V, \mathbf{n}_{a}, \boldsymbol{\nu , \varepsilon}}=\left(\frac{\partial Y_{j, \mathrm{off}}}{\partial \boldsymbol{\varepsilon}_{k}}\right)_{U, V, \mathbf{n}_{a}, \boldsymbol{\nu , \boldsymbol { \varepsilon }}},
$$

that is, the $c \times c$ matrix with elements $a_{j k}$ $=\left(\partial Y_{j, \text { off }} / \partial \varepsilon_{k}\right)_{U, V, \mathbf{n}_{a}, \boldsymbol{\nu}, \boldsymbol{\varepsilon}}$ is symmetric. Moreover, if we invert the relations

$$
Y_{k, \text { off }}=Y_{k, \text { off }}\left(U, V, \mathbf{n}_{a}+\boldsymbol{\nu} \cdot \boldsymbol{\varepsilon}\right) \text { for } k=1,2, \ldots, c,
$$

with respect to the variables $\varepsilon_{1}, \varepsilon_{2}, \ldots, \varepsilon_{c}$, we obtain the relations

$$
\varepsilon_{j}=\varepsilon_{j}\left(U, V, \mathbf{n}_{a}, \boldsymbol{\nu}, \mathbf{Y}_{\text {off }}\right) \text { for } j=1,2, \ldots, c,
$$

and using the properties of Jacobians we can easily show that the matrix with elements $b_{j k}=\left(\partial \varepsilon_{j} / \partial Y_{k, \text { off }}\right)_{U, V, \mathbf{n}_{a}, \boldsymbol{\nu}, \mathbf{Y}_{\text {off }}}$ is also symmetric; that is, for $j, k=1,2, \ldots, c$,

$$
\left(\frac{\partial \varepsilon_{k}}{\partial Y_{j, \text { off }}}\right)_{U, V, \mathbf{n}_{a}, \boldsymbol{\nu}, \mathbf{Y}_{\text {off }}}=\left(\frac{\partial \varepsilon_{j}}{\partial Y_{k, \text { off }}}\right)_{U, V, \mathbf{n}_{a}, \boldsymbol{\nu}, \mathbf{Y}_{\text {off }}}
$$

for both zero and nonzero values of $\mathbf{Y}_{\text {off }}$, that is, not only at the chemical equilibrium state of the reactor $A$, but also for any nonequilibrium state $A_{\varepsilon}$ that we model with the corresponding stable equilibrium state of the surrogate system $B$.

Relations (19) and (22) are among the many Maxwell relations that can be established for stable equilibrium states of a multiconstituent system, both for the surrogate system $B$ and the chemical equilibrium state of reactor $A$. Relation (22) implies that for the state of the surrogate system to remain in a stable equilibrium state and, hence, for the state of system $A$ to remain within the family $A_{\varepsilon}$, of the four changes $d \varepsilon_{j}$, $d \varepsilon_{k}, d Y_{j, \text { off }}$, and $d Y_{k, \text { off }}$, we can specify only three arbitrarily and independently.

\section{CONDITIONS FOR CHEMICAL EQUILIBRIUM IN TERMS OF TEMPERATURE AND PRESSURE}

Rather than using energy, volume, and amounts of constituents as independent variables, it is often more convenient to express each chemical equilibrium equation [Eqs. (16)] in terms of temperature, pressure, and mole fractions. To this end, we note that the stable equilibrium state of the surrogate system $B$ obtained by switching off the reaction mechanisms at a chemical equilibrium state of system $A$ has not only the same values of energy, entropy, volume, and amounts of constituents as the chemical equilibrium state, but also the same values of temperature, pressure, and chemical potentials $\mu_{1}, \mu_{2}, \ldots, \mu_{r}$.

For $i=1,2, \ldots, r$, to prove the last assertion, we recall the definitions of temperature, pressure, and chemical potentials ${ }^{4}$ as given, respectively, by the relations

$$
\begin{aligned}
& T\left(U, V, \mathbf{n}_{a} ; \boldsymbol{\nu}\right)=1 /(\partial S / \partial U)_{V, \mathbf{n}_{a}, \boldsymbol{\nu}}, \\
& p\left(U, V, \mathbf{n}_{a} ; \boldsymbol{\nu}\right)=(\partial S / \partial V)_{U, \mathbf{n}_{a}, \nu} /(\partial S / \partial U)_{V, \mathbf{n}_{a}, \boldsymbol{\nu}},
\end{aligned}
$$

$$
\mu_{i}\left(U, V, \mathbf{n}_{a} ; \boldsymbol{\nu}\right)=-\left(\partial S / \partial n_{i a}\right)_{U, V, \mathbf{n}_{a}, \boldsymbol{\nu}} /(\partial S / \partial U)_{V, \mathbf{n}_{a}, \boldsymbol{\nu}},
$$

where $S\left(U, V, \mathbf{n}_{a} ; \boldsymbol{\nu}\right)$ is the fundamental relation for the chemical equilibrium states [Eq. (8)].

Next, we express the fundamental relation $S$ of system $A$ in terms of that of the surrogate system $B$ by evaluating $S_{\text {off }}$ [Eq. (13)] at $\boldsymbol{\varepsilon}_{0}$ as given by Eq. (9), so that

$$
S=S\left(U, V, \mathbf{n}_{a} ; \boldsymbol{\nu}\right)=S_{\text {off }}\left(U, V, \mathbf{n}_{a}+\boldsymbol{\nu} \cdot \boldsymbol{\varepsilon}_{0}\left(U, V, \mathbf{n}_{a} ; \boldsymbol{\nu}\right)\right) .
$$

Thus, for the inverse temperature of a chemical equilibrium state, we find that 


$$
\begin{aligned}
& \frac{1}{T}=\frac{1}{T\left(U, V, \mathbf{n}_{a} ; \boldsymbol{\nu}\right)} \\
& =\left(\frac{\partial S}{\partial U}\right)_{V, \mathbf{n}_{a}, \boldsymbol{\nu}} \\
& =\left[\left(\frac{\partial S_{\mathrm{off}}}{\partial U}\right)_{V, \mathbf{n}}+\sum_{i=1}^{r}\left(\frac{\partial S_{\mathrm{off}}}{\partial n_{i}}\right)_{U, V, \mathbf{n}} \sum_{j=1}^{c} \nu_{i}^{(j)}\left(\frac{\partial \varepsilon_{j 0}}{\partial U}\right)_{V, \mathbf{n}_{a}, \nu}\right] \\
& =\left(\frac{\partial S_{\text {off }}}{\partial U}\right)_{V, \mathbf{n}}\left[1-\sum_{j=1}^{c}\left(\frac{\partial \varepsilon_{j 0}}{\partial U}\right)_{V, \mathbf{n}_{a}, \nu} \sum_{i=1}^{r} \nu_{i}^{(j)} \mu_{i, \mathrm{off}}\right] \\
& =\left(\frac{\partial S_{\mathrm{off}}}{\partial U}\right)_{V, \mathbf{n}} \\
& =\frac{1}{T_{\text {off }}\left(U, V, \mathbf{n}_{a}+\boldsymbol{\nu} \cdot \boldsymbol{\varepsilon}_{0}\left(U, V, \mathbf{n}_{a} ; \boldsymbol{\nu}\right)\right)}=\frac{1}{T_{\text {off }}},
\end{aligned}
$$

where in writing Eqs. (24c) and (24e) we use Eq. (23) and the chemical equilibrium equations [Eqs. (16)], respectively. So the temperature $T$ of a chemical equilibrium state equals the temperature $T_{\text {off }}$ of the corresponding state of the surrogate system $B$.

For the pressure of the chemical equilibrium state we find that

$$
\begin{aligned}
p & =p\left(U, V, \mathbf{n}_{a} ; \boldsymbol{\nu}\right) \\
& =T\left(\frac{\partial S}{\partial V}\right)_{U, \mathbf{n}_{a}, \boldsymbol{\nu}} \\
& =T\left[\left(\frac{\partial S_{\text {off }}}{\partial V}\right)_{U, \mathbf{n}}+\sum_{i=1}^{r}\left(\frac{\partial S_{\text {off }}}{\partial n_{i}}\right)_{U, V, \mathbf{n}} \sum_{j=1}^{c} \nu_{i}^{(j)}\left(\frac{\partial \varepsilon_{j 0}}{\partial V}\right)_{U, \mathbf{n}_{a}, \nu}\right] \\
& =T\left(\frac{\partial S_{\text {off }}}{\partial V}\right)_{U, \mathbf{n}}-\sum_{j=1}^{c}\left(\frac{\partial \varepsilon_{j 0}}{\partial V}\right)_{U, \mathbf{n}_{a}, \nu} \sum_{i=1}^{r} \nu_{i}^{(j)} \mu_{i, \text { off }} \\
& =T_{\text {off }}\left(\frac{\partial S_{\text {off }}}{\partial V}\right)_{U, \mathbf{n}} \\
& =p_{\text {off }}\left(U, V, \mathbf{n}_{a}+\boldsymbol{\nu} \cdot \boldsymbol{\varepsilon}_{0}\left(U, V, \mathbf{n}_{a} ; \boldsymbol{\nu}\right)\right)=p_{\text {off }},
\end{aligned}
$$

where in writing Eq. (25e) we use Eqs. (16) and (24). So the pressure $p$ of a chemical equilibrium state equals the pressure $p_{\text {off }}$ of the corresponding state of the surrogate system $B$.

For each chemical potential of the chemical equilibrium state we find

$$
\begin{aligned}
& \mu_{i}=\mu_{i}\left(U, V, \mathbf{n}_{a} ; \boldsymbol{\nu}\right) \\
& =-T\left(\frac{\partial S}{\partial n_{i a}}\right)_{U, V, \mathbf{n}_{a}, \nu} \\
& =-T\left[\left(\frac{\partial S_{\text {off }}}{\partial n_{i}}\right)_{U, V, \mathbf{n}}+\sum_{k=1}^{r}\left(\frac{\partial S_{\text {off }}}{\partial n_{k}}\right)_{U, V, \mathbf{n}}\right. \\
& \left.\times \sum_{j=1}^{c} \nu_{k}^{(j)}\left(\frac{\partial \boldsymbol{\varepsilon}_{j 0}}{\partial n_{i a}}\right)_{U, V, \mathbf{n}_{a}, \nu}\right] \\
& =-T\left(\frac{\partial S_{\mathrm{off}}}{\partial n_{i}}\right)_{U, V, \mathbf{n}}+\sum_{j=1}^{c}\left(\frac{\partial \varepsilon_{j 0}}{\partial n_{i a}}\right)_{U, V, \mathbf{n}_{a}, \nu} \sum_{k=1}^{r} \nu_{k}^{(j)} \mu_{k, \mathrm{off}} \\
& =-T_{\mathrm{off}}\left(\frac{\partial S_{\mathrm{off}}}{\partial n_{i}}\right)_{U, V, \mathbf{n}} \\
& =\mu_{i, \mathrm{off}}\left(U, V, \mathbf{n}_{a}+\boldsymbol{\nu} \cdot \boldsymbol{\varepsilon}_{0}\left(U, V, \mathbf{n}_{a} ; \boldsymbol{\nu}\right)\right)=\mu_{i, \mathrm{off}},
\end{aligned}
$$

where in writing Eq. (26e) we use Eqs. (16) and (24). So the chemical potential $\mu_{i}$ of the $i$ th constituent of a chemical equilibrium state of system $A$ equals the chemical potential $\mu_{i \text {,off }}$ of the $i$ th constituent of the corresponding state of the surrogate system $B$.

It is noteworthy that the identity of values of temperature, pressure, and chemical potentials of a chemical equilibrium state with the values of the respective properties of a stable equilibrium state of the surrogate system obtains only at chemical equilibrium, because then and only then are the chemical equilibrium equations [Eqs. (16)] satisfied. Away from chemical equilibrium states, temperature, pressure, and chemical potentials are not defined for system $A$ because all such states are not stable equilibrium.

Finally, we note that Eqs. (24)-(26) indicate that, geometrically, the surfaces represented by the functions $S$ $=S\left(U, V, \mathbf{n}_{a} ; \boldsymbol{\nu}\right)$ and $S_{\text {off }}=S_{\text {off }}\left(U, V, \mathbf{n}_{a}+\boldsymbol{\nu} \cdot \boldsymbol{\varepsilon}\right)$ have a contact of first degree for each given set of values $U, V$, and $\mathbf{n}_{a}$, at $\boldsymbol{\varepsilon}=\boldsymbol{\varepsilon}_{0}\left(U, V, \mathbf{n}_{a} ; \boldsymbol{\nu}\right)$, namely, at each chemical equilibrium state.

As is very well known, ${ }^{4}$ each chemical potential of a multiconstituent system in which all chemical reaction mechanisms are switched off may be expressed in the form $\mu_{i, \text { off }}=\mu_{i, \text { off }}\left(T, p, y_{1}, y_{2}, \ldots, y_{r}\right)$, where $y_{i}$ is the mole fraction of the $i$ th constituent. Using the stoichiometric relations and the chemical equilibrium equations, we find

$$
y_{i}=y_{i}\left(\mathbf{n}_{a}+\boldsymbol{\nu} \cdot \boldsymbol{\varepsilon}\right)=\frac{n_{i a}+\sum_{j=1}^{c} \nu_{i}^{(j)} \varepsilon_{j}}{n_{a}+\sum_{j=1}^{c} \nu^{(j)} \varepsilon_{j}}
$$

and, for $j=1,2, \ldots, c$,

$$
\begin{aligned}
\sum_{i=1}^{r} \nu_{i}^{(j)} \mu_{i, \mathrm{off}}\left(T, p, \frac{n_{1 a}+\sum_{j=1}^{c} \nu_{1}^{(j)} \varepsilon_{j 0}}{n_{a}+\sum_{j=1}^{c} \nu^{(j)} \varepsilon_{j 0}}, \ldots,\right. \\
\left.\ldots, \frac{n_{r a}+\sum_{j=1}^{c} \nu_{r}^{(j)} \varepsilon_{j 0}}{n_{a}+\sum_{j=1}^{c} \nu^{(j)} \varepsilon_{j 0}}\right)=-T Y_{j, \mathrm{off}}\left(T, p, \mathbf{y}_{0}\right)=0,
\end{aligned}
$$


where $n_{a}=\sum_{i=1}^{r} n_{i a}, \nu^{(j)}=\sum_{i=1}^{r} \nu_{i}^{(j)}$, and $y_{i 0}=y_{i}\left(\mathbf{n}_{a}+\boldsymbol{\nu} \cdot \boldsymbol{\varepsilon}_{0}\right)$.

Equations (28) represent the chemical equilibrium equations as functions of $T, p$, and the mole fractions of the chemical equilibrium state. The functional dependences of the chemical potentials on $T, p$, and $\mathbf{y}$ are those of surrogate system $B$. For given values of $T, p$, and $n_{a}$, we can solve the $c$ equations (28) for the $c$ unknowns $\varepsilon_{10}, \varepsilon_{20}, \ldots, \varepsilon_{c 0}$ and hence determine the chemical equilibrium composition $y_{10}, y_{20}, \ldots, y_{r 0}$ and the values of the corresponding amounts of constituents, $n_{10}, n_{20}, \ldots, n_{r 0}$. Conversely, if the values of $T, p, y_{1}, y_{2}, \ldots, y_{r}$ are given but do not satisfy Eqs. (28), we would conclude that the state is not chemical equilibrium. Then, of course, the values of $T$ and $p$ refer to a state of the surrogate system which becomes a nonequilibrium state of reactor $A$ when the reactions are turned on.

If the chemical potentials are written as

$$
\mu_{i, \mathrm{off}}(T, p, \mathbf{y})=\mu_{i i}(T, p)+R T \ln a_{i, \mathrm{off}}(T, p, \mathbf{y}),
$$

where $\mu_{i i}(T, p)$ is the pure constituent chemical potential at $T$ and $p, R$ the gas constant, and $a_{i, \text { off }}=a_{i, \text { off }}(T, p, \mathbf{y})$ the activity of the $i$ th constituent of the surrogate system, it is easy to see that we can rewrite the affinities as

$$
\begin{aligned}
\mathcal{A}_{j, \mathrm{off}} & =T Y_{j, \mathrm{off}} \\
& =-\sum_{i}^{r} \nu_{i}^{(j)} \mu_{i i}(T, p)-R T \ln \prod_{i=1}^{r}\left[a_{i, \mathrm{off}}\right]^{\nu_{i}^{(j)}},
\end{aligned}
$$

and, defining the equilibrium "constant" at temperature $T$ and pressure $p$ for the $j$ th reaction as

$$
K_{j}(T, p)=\exp \left(-\frac{1}{R T} \sum_{i}^{r} \nu_{i}^{(j)} \mu_{i i}(T, p)\right)
$$

we can write

$$
\prod_{i=1}^{r}\left[a_{i, \mathrm{off}}\right]_{i}^{(j)}=K_{j}(T, p) \exp \left(-Y_{j, \mathrm{off}} / R\right)
$$

and rewrite the chemical equilibrium equations (28) in the well-known mass-action-law form

$$
\prod_{i=1}^{r}\left[a_{i, \mathrm{off}}\left(T, p, \mathbf{y}_{0}\right)\right]^{\nu_{i}^{(j)}}=K_{j}(T, p) .
$$

Another interesting result is that the lowest value of the Gibbs free energy of the surrogate system $B$ obtains at the state of $B$ that corresponds to the chemical equilibrium state of $A$. Indeed, the Gibbs free energy of the surrogate system $B$ is

$$
G_{\text {off }}=G_{\text {off }}\left(T, p, n_{1}, n_{2}, \ldots, n_{r}\right) .
$$

If the amounts of constituents are compatible with $\mathbf{n}_{a}$ and the $c$ chemical reactions conform to Eqs. (12), we can rewrite $G_{\text {off }}$ in the form $G_{\text {off }}\left(T, p, \mathbf{n}_{a}+\boldsymbol{\nu} \cdot \boldsymbol{\varepsilon}\right)$. For given $T, p, \mathbf{n}_{a}$, and $\boldsymbol{\nu}$, an extreme value of $G_{\text {off }}$ obtains provided that, for each $j=1,2, \ldots, c$,

$$
\begin{aligned}
\left(\frac{\partial G_{\mathrm{off}}}{\partial \varepsilon_{j}}\right)_{T, p, \mathbf{n}_{a}, \boldsymbol{\nu}, \boldsymbol{\varepsilon}} & =\sum_{i=1}^{r}\left(\frac{\partial G_{\mathrm{off}}}{\partial n_{i}}\right)_{T, p, \mathbf{n}}\left(\frac{\partial n_{i}}{\partial \boldsymbol{\varepsilon}_{j}}\right)_{\mathbf{n}_{a}, \boldsymbol{\nu}, \boldsymbol{\varepsilon}} \\
& =\sum_{i=1}^{r} \mu_{i, \mathrm{off}} \nu_{i}^{(j)} \\
& =-T Y_{j, \mathrm{off}}\left(T, p, \mathbf{n}_{a}+\boldsymbol{\nu} \cdot \boldsymbol{\varepsilon}\right)=0
\end{aligned}
$$

where we use the equations $\left(\partial G / \partial n_{i}\right)_{T, p, \mathbf{n}}=\mu_{i}$ for $i$ $=1,2, \ldots, r$. These conditions are satisfied if the stable equilibrium state of the surrogate system corresponds to the chemical equilibrium state of $A$-that is, if the chemical potentials satisfy the chemical equilibrium equations [Eqs. (16)]. The extreme value of $G_{\text {off }}$ is a relative minimum because it can be shown that the second order partial differential of $G_{\text {off }}$ with respect to each of the $\varepsilon_{j}$ 's is positive.

By equating second order derivatives of $G_{\text {off }}$ with respect to the $\varepsilon_{j}$ 's, for $j, k=1,2, \ldots, c$,

$$
\frac{\partial^{2} G_{\mathrm{off}}\left(T, p, \mathbf{n}_{a}+\boldsymbol{\nu} \cdot \boldsymbol{\varepsilon}\right)}{\partial \varepsilon_{j} \partial \varepsilon_{k}}=\frac{\partial^{2} G_{\mathrm{off}}\left(T, p, \mathbf{n}_{a}+\boldsymbol{\nu} \cdot \boldsymbol{\varepsilon}\right)}{\partial \varepsilon_{k} \partial \varepsilon_{j}},
$$

we obtain the Maxwell relations

$$
\left(\frac{\partial Y_{k, \mathrm{off}}}{\partial \varepsilon_{j}}\right)_{T, p, \mathbf{n}_{a}, \boldsymbol{\nu}, \boldsymbol{\varepsilon}}=\left(\frac{\partial Y_{j, \mathrm{off}}}{\partial \varepsilon_{k}}\right)_{T, p, \mathbf{n}_{a}, \boldsymbol{\nu}, \boldsymbol{\varepsilon}} .
$$

Moreover, inverting the set of relations $Y_{k, \text { off }}$ $=Y_{k, \text { off }}\left(T, p, \mathbf{n}_{a}+\boldsymbol{\nu} \cdot \boldsymbol{\varepsilon}\right)$ for $k=1,2, \ldots, c$, with respect to the variables $\varepsilon_{1}, \varepsilon_{2}, \ldots, \varepsilon_{c}$, we obtain the set of relations $\varepsilon_{j}$ $=\varepsilon_{j}\left(T, p, \mathbf{n}_{a}, \boldsymbol{\nu}, \mathbf{Y}_{\text {off }}\right)$ for $j=1,2, \ldots, c$, and using the properties of Jacobians, we obtain the Maxwell relations

$$
\left(\frac{\partial \varepsilon_{k}}{\partial Y_{j, \mathrm{off}}}\right)_{T, p, \mathbf{n}_{a}, \boldsymbol{\nu}, \mathbf{Y}_{\mathrm{off}}}=\left(\frac{\partial \varepsilon_{j}}{\partial Y_{k, \mathrm{off}}}\right)_{T, p, \mathbf{n}_{a}, \boldsymbol{\nu}, \mathbf{Y}_{\mathrm{off}}}
$$

for both zero and nonzero values of $\mathbf{Y}_{\text {off }}$, that is, not only at the chemical equilibrium state of the reactor $A$, but also for any nonequilibrium state $A_{\varepsilon}$ that we model with the corresponding stable equilibrium state of the surrogate system $B$. Relations (37) and (38) can also be viewed as direct consequences of the Maxwell relations for the surrogate system obtained by equating second order derivatives of $G_{\text {off }}$ with respect to the $n_{j}$ 's,

$$
\left(\frac{\partial \mu_{k, \mathrm{off}}}{\partial n_{j}}\right)_{T, p, \mathbf{n}}=\left(\frac{\partial \mu_{j, \mathrm{off}}}{\partial n_{k}}\right)_{T, p, \mathbf{n}},
$$

or, equivalently,

$$
\left(\frac{\partial \ln a_{k, \mathrm{off}}}{\partial n_{j}}\right)_{T, p, \mathbf{n}}=\left(\frac{\partial \ln a_{j, \mathrm{off}}}{\partial n_{k}}\right)_{T, p, \mathbf{n}} .
$$

We conclude our derivation by summarizing the results pictorially with the help of the energy versus entropy graphs introduced in Ref. 4. For given values of $V$ and $\mathbf{n}_{a}$, three projections of states are superimposed on the single $U$ versus $S$ diagram shown in Fig. 1: (1) the projection of the states $A_{\varepsilon_{1}}$ of system $A$ that coincide with the stable equilibrium states of the surrogate system $B$ for the given volume $V$ of the chemical reactor and fixed values $\mathbf{n}_{1}=\mathbf{n}_{a}+\boldsymbol{\nu} \cdot \boldsymbol{\varepsilon}_{1}$, (2) the 


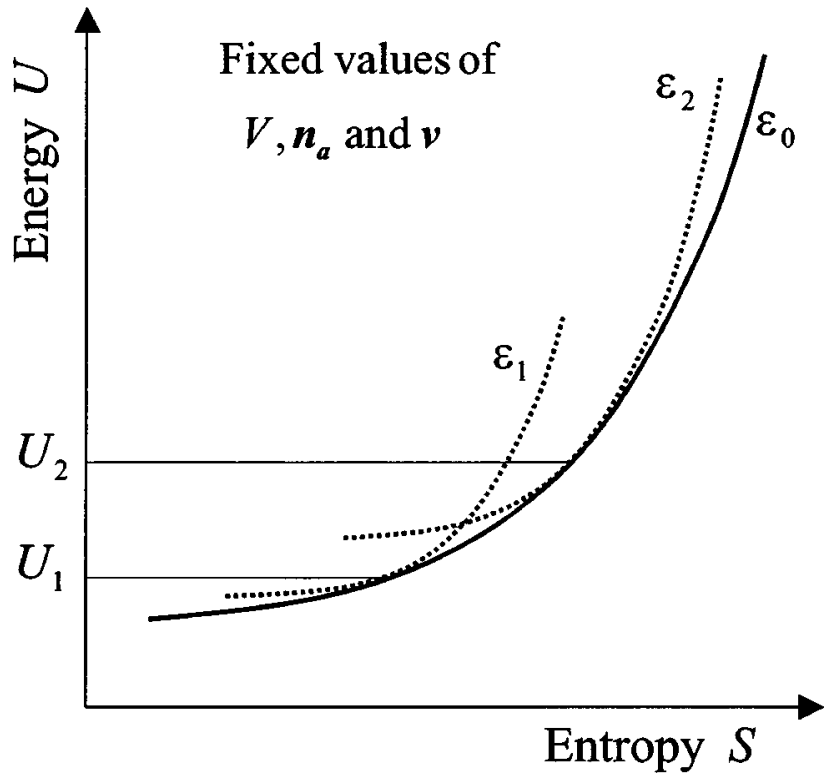

FIG. 1. Energy versus entropy graph of the states of simple system $A$ with given values of $V, \mathbf{n}_{a}$, and $\boldsymbol{\nu}$. The curve labeled $\varepsilon_{1}$ represents the states $A_{\varepsilon_{1}}$ and coincides with the curve of the stable equilibrium states of surrogate system $B$ for the given value $V$ of the volume and fixed values $\mathbf{n}_{1}=\mathbf{n}_{a}+\boldsymbol{\nu} \cdot \boldsymbol{\varepsilon}_{1}$ of the amounts of constituents-that is, a fixed $\boldsymbol{\varepsilon}_{1}$. The curve labeled $\varepsilon_{2}$ represents the states $A_{\varepsilon_{2}}$ and coincides with the curve of the stable equilibrium states of surrogate system $B$ for the given value of $V$ of the volume and fixed values $\mathbf{n}_{2}=\mathbf{n}_{a}+\boldsymbol{\nu} \cdot \boldsymbol{\varepsilon}_{2}$ of the amounts of constituentsthat is, a fixed $\varepsilon_{2}$. The curve labeled $\varepsilon_{0}$ represents the chemical equilibrium states of system $A$ for the given values of $V, \mathbf{n}_{a}$, and $\boldsymbol{\nu}$.

projection of the states $A_{\varepsilon_{2}}$ of system $A$ that coincide with the stable equilibrium states of the surrogate equation $B$ for the same given volume $V$ and fixed values $\mathbf{n}_{2}=\mathbf{n}_{a}+\boldsymbol{\nu} \cdot \boldsymbol{\varepsilon}_{2}$, and (3) the projection of the chemical equilibrium states of system $A$ for the same given values of $V, \mathbf{n}_{a}$, and $\boldsymbol{\nu}$.

The set of values $\boldsymbol{\varepsilon}_{1}$ is chosen so that $\boldsymbol{\varepsilon}_{1}$ $=\boldsymbol{\varepsilon}_{0}\left(U_{1}, V, \mathbf{n}_{a} ; \boldsymbol{\nu}\right)$ and, therefore, at the energy $U_{1}$ the locus of states $A_{\varepsilon_{1}}$ is tangent to the curve of the chemical equilibrium states of $A$. Similarly, the value $\boldsymbol{\varepsilon}_{2}$ is such that $\boldsymbol{\varepsilon}_{2}$ $=\boldsymbol{\varepsilon}_{0}\left(U_{2}, V, \mathbf{n}_{a} ; \boldsymbol{\nu}\right)$ and, therefore, the locus of states $A_{\boldsymbol{\varepsilon}_{2}}$ is tangent to the curve of the chemical equilibrium states of $A$ at the energy $U_{2}$ of the chemical reactor. We see that the curve of the chemical equilibrium states is the envelope of the loci of states $A_{\varepsilon}$ for all possible values of $\varepsilon$. We also see that the states $A_{\varepsilon_{1}}$ represent states of system $A$ that are not stable equilibrium, except at the energy $U_{1}$, and similarly the states $A_{\varepsilon_{2}}$, except at energy $U_{2}$. In general, they are either nonequilibrium or nonstable equilibrium states and yet can be described using the stable equilibrium properties of the surrogate system.

\section{RATE OF ENTROPY GENERATION}

In the course of chemical reactions in an isolated system $A$ with $r$ constituents and $c$ chemical reaction mechanisms, the system passes through a sequence of nonequilibrium states, and entropy is generated until the system reaches chemical equilibrium. At chemical equilibrium, all changes cease-the rate of change of each reaction coordinate is zero-and thereafter the system remains in the stable equilibrium state.

The rigorous and complete evaluation of the evolutions of the properties of the system as functions of time from any state that is not stable equilibrium towards the corresponding chemical or stable equilibrium state, and therefore the rate of entropy generation in a general nonequilibrium state is outside the scope of this article, and we are not discussing it.

Instead, nevertheless, we derive an estimate of the rate of entropy generation in terms of the $c$ affinities of the surrogate system $B$ of $A$ and the rates of change of the reaction coordinates of the $c$ chemical reaction mechanisms. We will see that this estimate is informative both about what might be considered as the driving forces of the reactions and about whether the so-called principle of microscopic reversibility plays any role in entropy generation. To derive this estimate, we proceed as follows.

For given values $U, V, \mathbf{n}_{a}$, and $\boldsymbol{\nu}$, the values of the amounts of constituents $\mathbf{n}=\mathbf{n}(t)=\left\{n_{1}, n_{2}, \ldots, n_{r}\right\}$, and the value of the entropy $S(t)$ are functions of time. Specifically, the value of $S(t)$ is smaller than the value of $S_{\text {off }}(U, V, \mathbf{n}(t))$ of the surrogate system $B$; that is,

$$
S(t) \leqslant S_{\text {off }}(U, V, \mathbf{n}(t))=S_{\text {off }}\left(U, V, \mathbf{n}_{a}+\boldsymbol{\nu} \cdot \boldsymbol{\varepsilon}(t)\right),
$$

where the equal sign applies only at the chemical equilibrium state. The justification of Inequality (40) is that, by definition, $S_{\text {off }}(U, V, \mathbf{n}(t))$ corresponds to the entropy of a stable equilibrium state which, by virtue of the largest entropy principle, is higher than the entropy of any other state with the same values of $U, V$, and $\mathbf{n}(t)$. Because at chemical equilibrium both $S(t)$ and $S_{\text {off }}(U, V, \mathbf{n}(t))$ assume the same largest value, an estimate of the rate of entropy generation $\dot{S}_{\text {irr }}$-the rate of entropy generation by irreversibility in the isolated system $A$-is obtained by assuming that $A$ is always in one of the states $A_{\varepsilon}$ defined in Sec. III, so that the value of each property is equal to the corresponding stable equilibrium state value of the surrogate system $B$.

This assumption corresponds to the following two-step conceptual model. We start at time $t$ with the surrogate system $B$ in a stable equilibrium state $B_{t, \text { eq }}$ corresponding to the state $A_{\boldsymbol{\varepsilon}(t)}$ of the reactor with reaction coordinates $\boldsymbol{\varepsilon}(t)$. We then turn the reactions on for an infinitesimal lapse of time $d t$ at the end of which we immediately turn them off. As a result, the reactor is in state $A_{t+d t}$, the reactions have changed the composition, and, in general, the surrogate system at time $t+d t$ is in a nonequilibrium state $B_{t+d t}$. We allow the additional lapse of time $\delta t^{\prime}$ that is necessary for the surrogate system to reach the stable equilibrium state $B_{t+d t+\delta t^{\prime}, \text { eq }}$ corresponding to the state $A_{\boldsymbol{\varepsilon}\left(t+d t+\delta t^{\prime}\right)}$ of the reactor with the new composition. Now we turn on the reactions again, and so on.

The values of the entropy are as follows: $S_{t, \mathrm{eq}}^{B}=S_{\boldsymbol{\varepsilon}(t)}^{A}$ and $S_{t+d t}^{B}=S_{t+d t}^{A}<S_{t+d t+\delta t^{\prime}, \text { eq }}^{B}=S_{\varepsilon\left(t+d t+\delta t^{\prime}\right)}^{A}$. The entropy generated by irreversibility in the first time interval is $S_{\text {irr, }(t, t+d t)}^{A}=S_{t+d t}^{A}-S_{\varepsilon(t)}^{A} \quad$ and in the second $S_{\text {irr, }\left(t+d t, t+d t+\delta t^{\prime}\right)}^{B}=S_{\varepsilon\left(t+d t+\delta t^{\prime}\right)}^{A}-S_{t+d t}^{A}$. An important simplification is obtained if $\delta t^{\prime} \ll d t$, that is, if the time $\delta t^{\prime}$ 


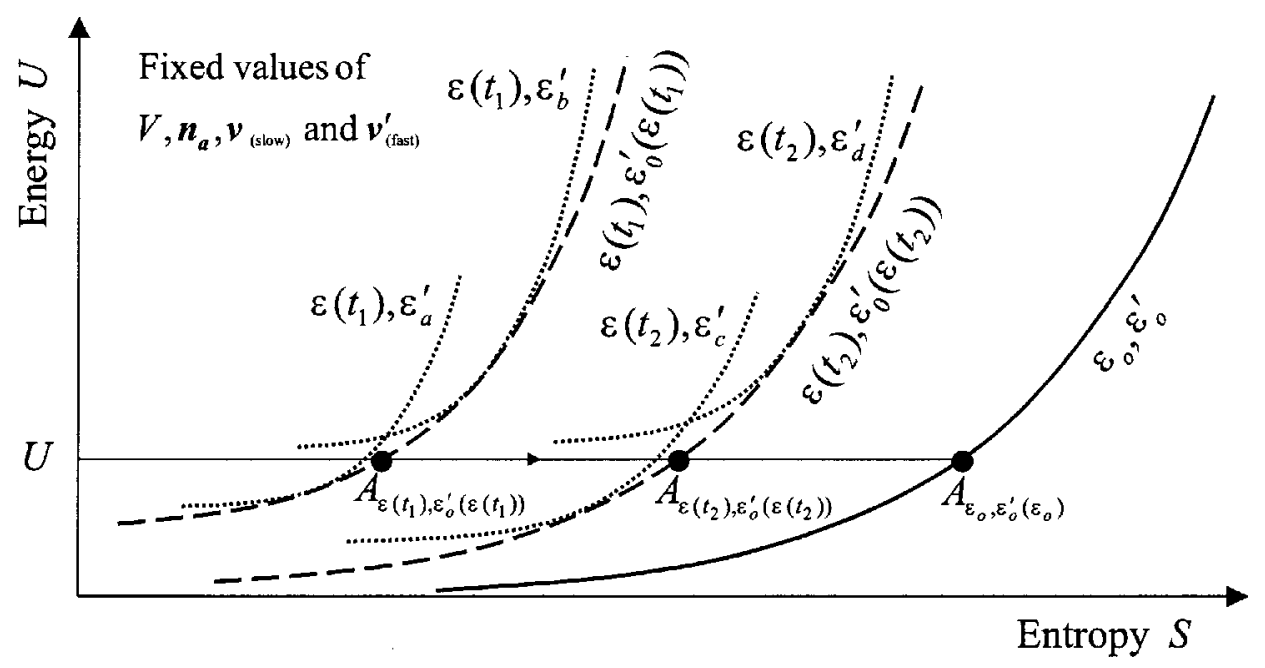

FIG. 2. Energy vs entropy graphs of the states of simple system $A$ with given values of $V, \mathbf{n}_{a}, \boldsymbol{\nu}$, and $\boldsymbol{\nu}^{\prime}$. State $A_{\boldsymbol{\varepsilon}(t), \boldsymbol{\varepsilon}_{0}^{\prime}(\varepsilon(t))}$ evolves in time while remaining on the dashed curve labeled $\boldsymbol{\varepsilon}(t), \boldsymbol{\varepsilon}_{0}^{\prime}(\boldsymbol{\varepsilon}(t))$ corresponding to the largest entropy (partial chemical equilibrium) state compatible with the given values $U, V$, $\mathbf{n}_{a}$, $\boldsymbol{\nu}, \boldsymbol{\nu}^{\prime}$ and the instantaneous values of reaction coordinates $\boldsymbol{\varepsilon}(t)$ of the slow rate-controlling reactions. The dotted curves represent the families of states $A_{\boldsymbol{\varepsilon}\left(t_{1}\right), \boldsymbol{\varepsilon}_{a}^{\prime}}, A_{\boldsymbol{\varepsilon}\left(t_{1}\right), \boldsymbol{\varepsilon}_{b}^{\prime}}, A_{\boldsymbol{\varepsilon}\left(t_{2}\right), \boldsymbol{\varepsilon}_{c}^{\prime}}, A_{\boldsymbol{\varepsilon}\left(t_{2}\right), \boldsymbol{\varepsilon}_{d}^{\prime}}$ for arbitrary values $\boldsymbol{\varepsilon}_{a}^{\prime}, \boldsymbol{\varepsilon}_{b}^{\prime}, \boldsymbol{\varepsilon}_{c}^{\prime}, \boldsymbol{\varepsilon}_{d}^{\prime}$. The solid curve labeled $\boldsymbol{\varepsilon}_{0}, \boldsymbol{\varepsilon}_{0}^{\prime}$ represents the chemical equilibrium states of system $A$ for the given values of $U, V, \mathbf{n}_{a}, \boldsymbol{\nu}$, and $\boldsymbol{\nu}^{\prime}$, where $\varepsilon_{0}^{\prime}=\varepsilon_{0}^{\prime}\left(\varepsilon_{0}\right)$.

taken by the surrogate system to reach stable equilibrium after a change in composition is much shorter than the time $d t$ taken by the reactor to affect such change in composition. In such a case, the second step of our conceptual model can be neglected, and the stable equilibrium states of the surrogate system are sufficient to describe the states of the reactor along the entire process.

This is tantamount to assuming that among all the internal interactions and dynamical mechanisms that drive the nonequilibrium state towards stable equilibrium, the chemical reactions with stoichiometric coefficients $\boldsymbol{\nu}$ are the only mechanisms capable of changing the composition of the system and, on the time scale chosen for the description, they are the slow, rate-controlling mechanisms, while all the other mechanisms are assumed to be much faster, so as to drive the system in negligible time to its largest entropy state compatible with the instantaneous values of $\mathbf{n}(t)$ and therefore maintain the state of the reactor along the family of states $A_{\varepsilon(t)}$ such that if at any instant in time we turn off the reactions we obtain the surrogate system at (or very close to) the stable equilibrium state with entropy $S_{\text {off }}\left(U, V, \mathbf{n}_{a}+\boldsymbol{\nu} \cdot \boldsymbol{\varepsilon}(t)\right)$.

Under this assumption,

$$
\begin{aligned}
\dot{S}_{\mathrm{irr}} & =\frac{d S_{\mathrm{off}}\left(U, V, \mathbf{n}_{a}+\boldsymbol{\nu} \cdot \boldsymbol{\varepsilon}(t)\right)}{d t} \\
& =\sum_{i=1}^{r}\left(\frac{\partial S_{\mathrm{off}}}{\partial n_{i}}\right)_{U, V, \mathbf{n}}\left(\frac{\partial n_{i}}{\partial t}\right)_{\mathbf{n}_{a}, \boldsymbol{\nu}} \\
& =-\sum_{i=1}^{r} \frac{\mu_{i, \mathrm{off}}}{T_{\mathrm{off}}} \sum_{j=1}^{c} \nu_{i}^{(j)} \dot{\varepsilon}_{j}=\sum_{j=1}^{c} \sum_{i=1}^{r}\left(-\frac{\nu_{i}^{(j)} \mu_{i, \mathrm{off}}}{T_{\mathrm{off}}}\right) \dot{\varepsilon}_{j} \\
& =\sum_{j=1}^{c} \dot{\varepsilon}_{j} Y_{j, \mathrm{off}}=\dot{\boldsymbol{\varepsilon}} \cdot \mathbf{Y}_{\mathrm{off}}=\dot{\boldsymbol{\varepsilon}}(t) \cdot \mathbf{Y}_{\mathrm{off}}\left(U, V, \mathbf{n}_{a}+\boldsymbol{\nu} \cdot \boldsymbol{\varepsilon}(t)\right),
\end{aligned}
$$

where $\dot{\boldsymbol{\varepsilon}}$ is the row vector of the $c$ reaction rates $\dot{\varepsilon}_{1}, \dot{\varepsilon}_{2}, \ldots, \dot{\varepsilon}_{c}$ and $Y_{\text {off }}$ the column vector of the $c$ ratios $Y_{j, \text { off }}=\mathcal{A}_{j, \text { off }} / T_{\text {off }}$ for $j=1,2, \ldots, c$, where $\mathcal{A}_{j, \text { off }}=-\sum_{i=1}^{r} \nu_{i}^{(j)} \mu_{i \text {, off }}$ is the affinity of the $j$ th reaction evaluated at the stable equilibrium state of surrogate system $B$ with values $U, V, \mathbf{n}(t)=\mathbf{n}_{a}+\boldsymbol{\nu} \cdot \boldsymbol{\varepsilon}(t)$.

A further simplification is obtained if there is a subset of chemical reaction mechanisms that are much faster than the others. Then, only the slow reactions are rate controlling, whereas the fast reactions drive the system in negligible time to its largest entropy state compatible with the instantaneous composition $\mathbf{n}(t)$, which varies slowly as a result of the rates of the slow reactions. Denoting the stoichiometric coefficients and the coordinates of the slow reactions by $\boldsymbol{\nu}$ and $\boldsymbol{\varepsilon}$ and of the fast reactions by $\boldsymbol{\nu}^{\prime}$ and $\boldsymbol{\varepsilon}^{\prime}$, Eqs. (6) and (12) are replaced by

$$
\sum_{i=1}^{r} \nu_{i}^{(j)} A_{i}=0 \quad \text { for } j=1,2, \ldots, c
$$

and

$$
\begin{aligned}
& \sum_{i=1}^{r} \nu_{i}^{\prime(k)} A_{i}=0 \text { for } k=1,2, \ldots, c^{\prime}, \\
& n_{i}(t)=n_{i a}+\sum_{j=1}^{c} \nu_{i}^{(j)} \varepsilon_{j}(t)+\sum_{k=1}^{c^{\prime}} \nu_{i}^{\prime(k)} \varepsilon_{k}^{\prime}(t) .
\end{aligned}
$$

In Eq. (43) we assume that the $\varepsilon_{k}^{\prime}$, s adjust instantly to changes in composition induced by the slowly varying $\varepsilon_{j}$ 's so as to maintain system $A$ at the largest entropy (partial chemical equilibrium) state $A_{\varepsilon, \varepsilon_{0}^{\prime}}$ compatible with the given values of $U, V, \mathbf{n}_{a}, \boldsymbol{\nu}, \boldsymbol{\nu}^{\prime}$ and the instantaneous values of the $\varepsilon_{j}(t)$ 's (see Fig. 2), that is, for $k=1,2, \ldots, c^{\prime}$,

$$
\varepsilon_{k}^{\prime}(t)=\varepsilon_{k 0}^{\prime}\left(U, V, \mathbf{n}_{a}+\boldsymbol{\nu} \cdot \boldsymbol{\varepsilon}(t) ; \boldsymbol{\nu}^{\prime}\right),
$$


where the $\varepsilon_{k 0}^{\prime}$ 's are the values that maximize $S_{\boldsymbol{\varepsilon}, \boldsymbol{\varepsilon}^{\prime}}$ $=S_{\text {off }}\left(U, V, \mathbf{n}_{a}+\boldsymbol{\nu} \cdot \boldsymbol{\varepsilon}+\boldsymbol{\nu}^{\prime} \cdot \boldsymbol{\varepsilon}^{\prime}\right)$ for the given values of $U, V$, $\mathbf{n}_{a}, \boldsymbol{\nu}, \boldsymbol{\nu}^{\prime}$, and $\boldsymbol{\varepsilon}(t)$, or equivalently, for $k=1,2, \ldots, c^{\prime}$ :

$$
\begin{aligned}
& \left(\frac{\partial S_{\boldsymbol{\varepsilon}, \boldsymbol{\varepsilon}^{\prime}}}{\partial \varepsilon_{k}^{\prime}}\right)_{U, V, \mathbf{n}_{a}+\boldsymbol{\nu} \cdot \boldsymbol{\varepsilon}, \boldsymbol{\nu}^{\prime}, \boldsymbol{\varepsilon}^{\prime}} \\
& \quad=Y_{k, \mathrm{off}}^{\prime}\left(U, V, \mathbf{n}_{a}+\boldsymbol{\nu} \cdot \boldsymbol{\varepsilon}(t)+\boldsymbol{\nu}^{\prime} \cdot \boldsymbol{\varepsilon}_{0}^{\prime}\right)=0 .
\end{aligned}
$$

As a result of these assumptions, system $A$ evolves through the family of states $A_{\boldsymbol{\varepsilon}(t), \boldsymbol{\varepsilon}_{0}^{\prime}(\boldsymbol{\varepsilon}(t))}$, the entropy $S(t)$ is approximated in terms of $S_{\text {off }}$ of the surrogate system $B$,

$$
\begin{aligned}
S(t)= & S_{\mathrm{off}}\left(U, V, \mathbf{n}_{a}+\boldsymbol{\nu} \cdot \boldsymbol{\varepsilon}(t)\right. \\
& \left.+\boldsymbol{\nu}^{\prime} \cdot \boldsymbol{\varepsilon}_{0}^{\prime}\left(U, V, \mathbf{n}_{a}+\boldsymbol{\nu} \cdot \boldsymbol{\varepsilon}(t) ; \boldsymbol{\nu}^{\prime}\right)\right),
\end{aligned}
$$

and the rate of entropy generation is given by the relation

$$
\begin{aligned}
\dot{S}_{\mathrm{irr}}= & \sum_{j=1}^{c} \dot{\varepsilon}_{j} Y_{j, \mathrm{off}}+\sum_{k=1}^{c^{\prime}} \dot{\varepsilon}_{k 0}^{\prime} Y_{k, \mathrm{off}}^{\prime} \\
= & \sum_{j=1}^{c} \dot{\varepsilon}_{j}(t) Y_{j, \mathrm{off}}\left(U, V, \mathbf{n}_{a}+\boldsymbol{\nu} \cdot \boldsymbol{\varepsilon}(t)\right. \\
& \left.+\boldsymbol{\nu}^{\prime} \cdot \boldsymbol{\varepsilon}_{0}^{\prime}\left(U, V, \mathbf{n}_{a}+\boldsymbol{\nu} \cdot \boldsymbol{\varepsilon}(t) ; \boldsymbol{\nu}^{\prime}\right)\right),
\end{aligned}
$$

because at each instant of time $Y_{k, \text { off }}^{\prime}=0$ [Eq. (45)].

It is clear that the model we present here is valid for homogeneous states of the reactor. In addition, it provides the conceptual background also for the so-called local equilibrium assumption upon which the continuum fluid dynamics treatment of nonhomogeneous states is based.

\section{RECIPROCAL RELATIONS}

According to the foregoing discussion, we proceed under the assumption-approximation that the (homogeneous) state of the isolated reactor $A$ belongs at each instant in time to the family of states $A_{\varepsilon}$. For fixed values of $U, V, \mathbf{n}_{a}$, and $\boldsymbol{\nu}$, the only independent variables of the family of $A_{\varepsilon}$ states are the reaction coordinates $\varepsilon_{j}$ 's. We further assume that each reaction rate $\dot{\varepsilon}_{k}$ is a function of the state $A_{\varepsilon}$ and, hence, of the $\varepsilon_{j}$ 's, that is,

$$
\dot{\varepsilon}=\dot{\varepsilon}(\varepsilon)
$$

with

$$
\dot{\boldsymbol{\varepsilon}}\left(\boldsymbol{\varepsilon}_{0}\right)=\mathbf{0},
$$

where, for simplicity, from here on we do not write explicitly the dependences on the given values of $U, V, \mathbf{n}_{a}, \boldsymbol{\nu}$, and $\boldsymbol{\nu}^{\prime}$. Condition (49) is necessary because at chemical equilibrium all reaction rates are zero.

Recalling that $\mathbf{Y}_{\text {off }}=\mathbf{Y}_{\text {off }}(\boldsymbol{\varepsilon})$ [Eqs. (15)], we can write Eqs. (41) and (47) for the rate of entropy generation in the form

$$
\dot{S}_{\mathrm{irr}}=\dot{S}_{\mathrm{irr}}(\boldsymbol{\varepsilon})=\sum_{\ell=1}^{c} \dot{\varepsilon}_{\ell}(\boldsymbol{\varepsilon}) Y_{\ell, \mathrm{off}}(\boldsymbol{\varepsilon})=\dot{\boldsymbol{\varepsilon}}(\boldsymbol{\varepsilon}) \cdot \mathbf{Y}_{\mathrm{off}}(\boldsymbol{\varepsilon})
$$

and note that nowhere in our derivation we make reference to the concept of microscopic reversibility.

Next we make use of the condition that, at every state $A_{\varepsilon}, \dot{S}_{\text {irr }}(\boldsymbol{\varepsilon})$ must be non-negative. In particular, we apply this condition in the vicinity of the chemical equilibrium state by expanding $\dot{S}_{\text {irr }}(\boldsymbol{\varepsilon})$ into a Taylor series about $\boldsymbol{\varepsilon}_{0}$ up to second order and using the fact that $Y_{j, \text { off }}\left(\varepsilon_{0}\right)=0$ [Eq. (35)], $\dot{\varepsilon}_{j}\left(\boldsymbol{\varepsilon}_{0}\right)=0$ [Eq. (49)], and, of course, $\dot{S}_{\text {irr }}\left(\boldsymbol{\varepsilon}_{0}\right)=0$. Thus, for $j, k=1,2, \ldots, c$, we find

$$
\begin{aligned}
\left.\frac{\partial \dot{S}_{\mathrm{irr}}(\boldsymbol{\varepsilon})}{\partial \varepsilon_{j}}\right|_{\boldsymbol{\varepsilon}_{0}}= & \left.\sum_{\ell=1}^{c} \frac{\partial \dot{\boldsymbol{\varepsilon}}_{\ell}}{\partial \varepsilon_{j}}\right|_{\varepsilon_{0}} Y_{\ell, \mathrm{off}}\left(\boldsymbol{\varepsilon}_{0}\right) \\
& +\left.\sum_{\ell=1}^{c} \dot{\varepsilon}_{\ell}\left(\boldsymbol{\varepsilon}_{0}\right) \frac{\partial Y_{\ell, \mathrm{off}}}{\partial \boldsymbol{\varepsilon}_{j}}\right|_{\boldsymbol{\varepsilon}_{0}}=0,
\end{aligned}
$$

$$
\begin{aligned}
\left.\frac{\partial^{2} \dot{S}_{\text {irr }}(\boldsymbol{\varepsilon})}{\partial \varepsilon_{j} \partial \varepsilon_{k}}\right|_{\varepsilon_{0}}= & \left.\left.\sum_{\ell=1}^{c} \frac{\partial \dot{\varepsilon}_{\ell}}{\partial \varepsilon_{j}}\right|_{\varepsilon_{0}} \frac{\partial Y_{\ell, \text { off }}}{\partial \varepsilon_{k}}\right|_{\varepsilon_{0}} \\
& +\left.\left.\sum_{\ell=1}^{c} \frac{\partial \dot{\varepsilon}_{\ell}}{\partial \varepsilon_{k}}\right|_{\dot{\boldsymbol{\varepsilon}}_{0}} \frac{\partial Y_{\ell, \mathrm{off}}}{\partial \varepsilon_{j}}\right|_{\boldsymbol{\varepsilon}_{0}}
\end{aligned}
$$

and, therefore,

$$
\begin{aligned}
\dot{S}_{\mathrm{irr}}(\boldsymbol{\varepsilon})= & \left.\frac{1}{2} \sum_{j=1}^{c} \sum_{k=1}^{c} \frac{\partial^{2} \dot{S}_{\mathrm{irr}}(\boldsymbol{\varepsilon})}{\partial \varepsilon_{j} \partial \varepsilon_{k}}\right|_{\varepsilon_{0}}\left(\varepsilon_{j}-\varepsilon_{j 0}\right)\left(\varepsilon_{k}-\varepsilon_{k 0}\right)+\cdots \\
= & \left.\left.\sum_{j=1}^{c} \sum_{k=1}^{c} \sum_{\ell=1}^{c} \frac{\partial \dot{\varepsilon}_{\ell}}{\partial \varepsilon_{j}}\right|_{\varepsilon_{0}} \frac{\partial Y_{\ell, \mathrm{off}}}{\partial \varepsilon_{k}}\right|_{\varepsilon_{0}}\left(\varepsilon_{j}-\varepsilon_{j 0}\right)\left(\varepsilon_{k}-\varepsilon_{k 0}\right) \\
& +\cdots \\
= & \left.\left.\left.\sum_{j=1}^{c} \sum_{k=1}^{c} \sum_{\ell=1}^{c} \sum_{m=1}^{c} \frac{\partial \dot{\tilde{\varepsilon}}_{\ell}}{\partial Y_{m, \mathrm{off}}}\right|_{\boldsymbol{\varepsilon}_{0}} \frac{\partial Y_{m, \mathrm{off}}}{\partial \varepsilon_{j}}\right|_{\varepsilon_{0}} \frac{\partial Y_{\ell, \mathrm{off}}}{\partial \varepsilon_{k}}\right|_{\varepsilon_{0}} \\
& \times\left(\varepsilon_{j}-\varepsilon_{j 0}\right)\left(\varepsilon_{k}-\varepsilon_{k 0}\right)+\cdots \\
= & \left.\sum_{\ell=1}^{c} \sum_{m=1}^{c} \frac{\partial \dot{\tilde{\varepsilon}}_{\ell}}{\partial Y_{m, \mathrm{off}}}\right|_{\varepsilon_{0}} Y_{\ell, \mathrm{off}}(\boldsymbol{\varepsilon}) Y_{m, \mathrm{off}}(\boldsymbol{\varepsilon})+\cdots \\
= & \sum_{\ell=1}^{c} \sum_{m=1}^{c} L_{\ell m, \mathrm{off}} Y_{\ell, \mathrm{off}}(\boldsymbol{\varepsilon}) Y_{m, \mathrm{off}}(\boldsymbol{\varepsilon})+\cdots
\end{aligned}
$$

where we define the $c \times c$ matrix $\mathbf{L}$ with elements

$$
L_{\ell m, \mathrm{off}}=\left.\frac{\partial \dot{\tilde{\varepsilon}}_{\ell}}{\partial Y_{m, \mathrm{off}}}\right|_{\boldsymbol{\varepsilon}_{0}} .
$$

In Eq. (53c) we use the truncated Taylor series expansion about $\boldsymbol{\varepsilon}_{0}$ of the relations

$$
\dot{\boldsymbol{\varepsilon}}=\dot{\tilde{\boldsymbol{\varepsilon}}}\left(\mathbf{Y}_{\text {off }}\right)=\dot{\boldsymbol{\varepsilon}}\left(\boldsymbol{\varepsilon}\left(\mathbf{Y}_{\text {off }}\right)\right),
$$

which follow ${ }^{8}$ from substituting into Eq. (48) the relations $\boldsymbol{\varepsilon}=\boldsymbol{\varepsilon}\left(\mathbf{Y}_{\text {off }}\right)$ obtained from the inversion (at fixed $U, V, \mathbf{n}_{a}, \boldsymbol{\nu}$, and $\boldsymbol{\nu}^{\prime}$ ) of the set of relations $\mathbf{Y}_{\text {off }}=\mathbf{Y}_{\text {off }}(\boldsymbol{\varepsilon})$, that is, for $j, \ell$ $=1,2, \ldots, c$, 


$$
\begin{array}{r}
\dot{\tilde{\varepsilon}}_{\ell}\left(\mathbf{Y}_{\mathrm{off}}(\boldsymbol{\varepsilon})\right)=\left.\sum_{m=1}^{c} \frac{\partial \dot{\tilde{\varepsilon}}_{\ell}}{\partial Y_{m, \mathrm{off}}}\right|_{\boldsymbol{\varepsilon}_{0}} Y_{m, \mathrm{off}}(\boldsymbol{\varepsilon})+\cdots \\
=\sum_{m=1}^{c} L_{\ell m, \mathrm{off}} Y_{m, \mathrm{off}}(\boldsymbol{\varepsilon})+\cdots, \\
\left.\frac{\partial \dot{\boldsymbol{\varepsilon}}_{\ell}}{\partial \boldsymbol{\varepsilon}_{j}}\right|_{\boldsymbol{\varepsilon}_{0}}=\left.\left.\sum_{m=1}^{c} \frac{\partial \dot{\tilde{\varepsilon}}_{\ell}}{\partial Y_{m, \mathrm{off}}}\right|_{\boldsymbol{\varepsilon}_{0}} \frac{\partial Y_{m, \mathrm{off}}}{\partial \boldsymbol{\varepsilon}_{j}}\right|_{\boldsymbol{\varepsilon}_{0}}+\cdots
\end{array}
$$

In Eq. (53d) we use the truncated expansion about $\boldsymbol{\varepsilon}_{0}$ of the relations $\mathbf{Y}_{\text {off }}=\mathbf{Y}_{\text {off }}(\boldsymbol{\varepsilon})$, that is, for $\ell=1,2, \ldots, c$,

$$
Y_{m, \mathrm{off}}(\boldsymbol{\varepsilon})=\left.\sum_{j=1}^{c} \frac{\partial Y_{m, \mathrm{off}}}{\partial \boldsymbol{\varepsilon}_{j}}\right|_{\boldsymbol{\varepsilon}_{0}}\left(\varepsilon_{j}-\varepsilon_{j 0}\right)+\cdots
$$

Our results can be interpreted in the customary manner of the so-called "Onsager's linear thermodynamic theory of irreversible processes." $9-12$ Each affinity $\mathcal{A}_{m \text {,off }}$ or, better, each $Y_{m, \text { off }}=\mathcal{A}_{m \text {,off }} / T_{\text {off }}$ for $m=1,2, \ldots, c$, can be regarded as a "driving force" and each reaction rate $\dot{\varepsilon}_{j}$, for $j$ $=1,2, \ldots, c$, as a "flux" that depends on all the driving forces: that is, "driving forces" and "fluxes" are coupled. If the fluxes are expressed as functions of the driving forces or vice versa, the coefficients of the linear approximation [Eqs. (56)] of these functions in the vicinity of the chemical equilibrium state can be regarded as "generalized conductivities."

In view of the relations $\boldsymbol{\varepsilon}=\boldsymbol{\varepsilon}\left(\mathbf{Y}_{\text {off }}\right)$, with $\boldsymbol{\varepsilon}(\mathbf{0})=\boldsymbol{\varepsilon}_{0}$, we rewrite Eq. (50) in the form

$$
\dot{S}_{\mathrm{irr}}=\dot{S}_{\mathrm{irr}}\left(\mathbf{Y}_{\mathrm{off}}\right)=\sum_{\ell=1}^{c} \dot{\varepsilon}_{\ell}\left(\boldsymbol{\varepsilon}\left(\mathbf{Y}_{\mathrm{off}}\right)\right) Y_{\ell, \mathrm{off}}=\dot{\tilde{\varepsilon}}\left(\mathbf{Y}_{\mathrm{off}}\right) \cdot \mathbf{Y}_{\text {off }},
$$

where $\dot{\boldsymbol{\varepsilon}}=\dot{\tilde{\boldsymbol{\varepsilon}}}\left(\mathbf{Y}_{\text {off }}\right)=\dot{\boldsymbol{\varepsilon}}\left(\boldsymbol{\varepsilon}\left(\mathbf{Y}_{\text {off }}\right)\right)$ with $\dot{\tilde{\boldsymbol{\varepsilon}}}(\mathbf{0})=\dot{\boldsymbol{\varepsilon}}\left(\boldsymbol{\varepsilon}_{0}\right)=\mathbf{0}$. The expansion of this form in the vicinity of the chemical equilibrium state yields

$$
\dot{S}_{\text {irr }}\left(\mathbf{Y}_{\text {off }}\right)=\left.\frac{1}{2} \sum_{j=1}^{c} \sum_{k=1}^{c} \frac{\partial^{2} \dot{S}_{\text {irr }}\left(\mathbf{Y}_{\text {off }}\right)}{\partial Y_{j, \text { off }} \partial Y_{k, \text { off }}}\right|_{0} Y_{j, \text { off }} Y_{k, \text { off }}+\cdots
$$

Direct comparison of Eq. (60) with Eq. (53e) shows that, for $j, k=1,2, \ldots, c$,

$$
L_{j k, \text { off }}=\left.\frac{1}{2} \frac{\partial^{2} \dot{S}_{\text {irr }}\left(\mathbf{Y}_{\text {off }}\right)}{\partial Y_{j, \text { off }} \partial Y_{k, \text { off }}}\right|_{0}=\left.\frac{1}{2} \frac{\partial^{2} \dot{S}_{\text {irr }}\left(\mathbf{Y}_{\text {off }}\right)}{\partial Y_{k, \text { off }} \partial Y_{j, \text { off }}}\right|_{0}=L_{j k, \text { off }},
$$

and therefore the matrix $\mathbf{L}$ is symmetric, that is, its elements obey the Onsager ${ }^{10}$ reciprocal relations.

Equation (53a) shows that the leading term in the expansion of $\dot{S}_{\text {irr }}$ around the chemical equilibrium state is a quadratic form in the "distances" $\left(\varepsilon_{j}-\varepsilon_{j 0}\right)$ from the chemical equilibrium state. Because in the vicinity of the chemical equilibrium state every $\varepsilon_{j}-\varepsilon_{j 0}$ can take both negative and positive values, the condition that $\dot{S}_{\text {irr }}$ be always nonnegative implies that the coefficients of the quadratic form are elements of a nonnegative definite matrix, that is,

$$
\mathbf{S}_{\text {off }}^{\prime \prime} \geqslant 0 \text {, }
$$

where the elements of the $c \times c$ matrix $\mathbf{S}_{\text {off }}^{\prime \prime}$ are given by the relations

$$
S_{j k, \text { off }}^{\prime \prime}=\left.\frac{1}{2} \frac{\partial^{2} \dot{S}_{\text {irr }}(\boldsymbol{\varepsilon})}{\partial \varepsilon_{j} \partial \varepsilon_{k}}\right|_{\varepsilon_{0}}
$$

Similarly, Eq. (53e) shows that the leading term in the expansion of $\dot{S}_{\text {irr }}$ around the chemical equilibrium state is also a quadratic form in the "driving forces" $Y_{j, \text { off }}(\boldsymbol{\varepsilon})$. Because in the vicinity of the chemical equilibrium state every $Y_{j, \text { off }}(\boldsymbol{\varepsilon})$ can take both negative and positive values, the condition that $\dot{S}_{\text {irr }}$ be always non-negative implies that also the coefficients of this quadratic form are elements of a nonnegative definite matrix

$$
\mathbf{L}\left(\boldsymbol{\varepsilon}_{0}\right) \geqslant 0 \text {; }
$$

that is, the symmetric matrix of reciprocity or Onsager coefficients is non-negative definite.

We finally emphasize that the main results of this section derive from the structure of the leading terms of Taylor expansions valid only in the vicinity of the chemical equilibrium state.

By contrast, the model developed in the previous sections in terms of the $A_{\varepsilon}$ family of states and the stable equilibrium properties of the surrogate system is valid both far from and near the chemical equilibrium state. As it is well known, ${ }^{11}$ the relation $\dot{\boldsymbol{\varepsilon}}=\dot{\tilde{\boldsymbol{\varepsilon}}}\left(\mathbf{Y}_{\text {off }}\right)$ between reaction rates and driving forces is in general nonlinear. Nevertheless, even for states that are very far from chemical equilibrium, relations (50) and (59) are valid, together with the condition that $\dot{S}_{\text {irr }} \geqslant 0$, and provide important guidance in the development of chemical kinetics models.

\section{CONCLUSIONS}

The thermodynamic derivations of conditions for chemical equilibrium, of Onsager reciprocity relations, and of the properties of a practically important family of nonequilibrium states presented here differ from the derivations presented in practically all treatises of thermodynamics applied to chemical reactors.

Our motivation for developing this derivation is based on a "revolutionary" 13 conception of thermodynamics as a "nonstatistical" 14 physical theory ${ }^{4,15}$ that applies to all systems (both macroscopic and microscopic), to all states (both thermodynamic equilibrium and not thermodynamic equilibrium), and that discloses that entropy is an inherent (intrinsic) nondestructible property of matter (well defined for all systems and all states), in the same sense as inertial mass is an inherent property of matter.

Our nonstatistical derivation of Onsager relations for an isolated chemical reactor shows that the arguments based on statistical fluctuations, time reversal, and the principle of microscopic reversibility, ${ }^{9-12}$ which are invariably used in all traditional derivations, are not essential and, therefore, play no fundamental role in the thermodynamic theory of irreversible processes. 
${ }^{1}$ E. A. Guggenheim, Thermodynamics, 5th revised ed. (North-Holland, Amsterdam, 1967).

${ }^{2}$ K. Denbigh, The Principles of Chemical Equilibrium, 2nd ed. (Cambridge University Press, London, 1966).

${ }^{3}$ M. Modell and R. C. Reid, Thermodynamics and Its Applications (Prentice Hall, Englewood Cliffs, NJ, 1974).

${ }^{4}$ E. P. Gyftopoulos and G. P. Beretta, Thermodynamics: Foundations and Applications (Macmillan, New York, 1991).

${ }^{5}$ The simple system model extends to heterogeneous stable equilibrium states also in the presence of external fields and surface effects, provided a continuous phase model is adopted. See, e.g., J. W. Cahn and J. E. Hilliard, J. Chem. Phys. 28, 258 (1958); L. Mistura, ibid. 83, 3633 (1985); and Ref. 7.

${ }^{6}$ J. C. Slater, Quantum Theory of Molecules and Solids (McGraw-Hill, New York, 1965), Vol. 2.

${ }^{7}$ G. N. Hatsopoulos and E. P. Gyftopoulos, Thermionic Energy Conversion (MIT Press, Cambridge, MA, 1979), Vol. 2.

${ }^{8}$ For the sake of precision, we introduce the different character $\dot{\tilde{\varepsilon}}$ to em- phasize that, as defined by Eq. (55), the functional dependence implied by writing $\dot{\boldsymbol{\varepsilon}}=\dot{\tilde{\boldsymbol{\varepsilon}}}\left(\mathbf{Y}_{\text {off }}\right)$ is different from that implied by writing $\dot{\boldsymbol{\varepsilon}}=\dot{\boldsymbol{\varepsilon}}(\boldsymbol{\varepsilon})$.

${ }^{9}$ R. C. Tolman, The Principles of Statistical Mechanics (Oxford University Press, Oxford, 1938).

${ }^{10}$ L. Onsager, Phys. Rev. 37, 405 (1931); 38, 2265 (1931).

${ }^{11}$ S. R. deGroot and P. Mazur, Nonequilibrium Thermodynamics (NorthHolland, Amsterdam, 1962).

${ }^{12}$ A. Katchalsky and P. F. Curran, Nonequilibrium Thermodynamics in Biophysics (Harvard University Press, Cambridge, MA, 1967).

${ }^{13}$ We use the term "revolutionary" in the sense introduced by T. S. Kuhn, The Structure of Scientific Revolutions (The University of Chicago Press, Chicago, 1970).

${ }^{14}$ We use the term "nonstatistical" to emphasize that entropy is defined (see Refs. 4 and 15) for all systems, macroscopic and microscopic, and all states, thermodynamic equilibrium and not thermodynamic equilibrium, without any necessity for either statistical or information theoretic arguments.

${ }^{15}$ G. N. Hatsopoulos and E. P. Gyftopoulos, Found. Phys. 6, 15 (1976); 6, 127 (1976); 6, 439 (1976); 6, 561 (1976); G. P. Beretta, ibid. 17, 365 (1987). 